\title{
Zur Kenntnis des Uranminerals Trögerit
}

\author{
Von A. Weiss, F. Taborszky, K. Hartl und
}

E. TRÖGER

Aus dem Eduard Zintl-Institut für anorganische und physikalische Chemie der Technischen Hochschule Darmstadt und dem Mineralogischen Institut der Universität Freiburg

(Z. Naturforschg. 12 b, 356-358 [1957] ; eingegangen am 18. Februar 1957)

Das Uranmineral Trögerit wurde auf Grund seines Kationen-Austauschvermögens, seiner Fähigkeit zur eindimensionalen innerkristallinen Quellung, seiner chemischen Zusammensetzung, seiner Dichte und seiner Gitterdimensionen als Uranglimmer identifiziert. Die Strukturformel ergab sich zu:

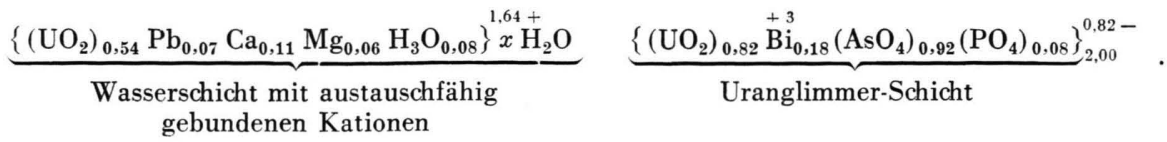

1,64 Äquivalente Kationen pro Formeleinheit sind austauschfähig gebunden, davon 1,08 Äquivalente Uranylionen.

Die Kristalle weisen starke Gitterstörungen auf. Ungestörte Kristalle zeigen z. T. Überstrukturen, die zu einer Vergrößerung der Elementarzelle gegenüber normalen Uranglimmern führen.

Unter Wasser ergab sich eine monokline Zelle mit

$$
\begin{aligned}
& a=7,1_{5} \AA, \\
& b=6,9_{9} \AA, \\
& c=11,2 \AA, \\
& \text { und } \quad \beta \approx 95^{\circ} .
\end{aligned}
$$

Die lufttrockenen Kristalle waren tetragonal mit

$$
\begin{aligned}
& a=13,9_{8} \AA=2 \cdot 6,9_{9} \AA \\
& \text { und } \quad c
\end{aligned}
$$

Die bei $210^{\circ} \mathrm{C}$ getrockneten Kristalle waren gleichfalls tetragonal mit

$$
\begin{aligned}
& \quad a=7,0_{4} \AA \text { A, } \\
& \text { und } \quad c=8,3_{9} \AA .
\end{aligned}
$$

Trögerit ${ }^{1}$ ist ein seltenes Uranmineral, dessen Struktur noch nicht gedeutet worden ist. MeIxner ${ }^{2}$ formulierte das Mineral als

$$
\mathrm{UO}_{2} \cdot\left(\mathrm{UO}_{2}\right)_{2} \cdot\left(\mathrm{AsO}_{4}\right)_{2} \cdot 12 \mathrm{H}_{2} \mathrm{O},
$$

während $\mathrm{Mrose}^{3}$ annahm, daß der Trögerit mit künstlichem Oxonium-Uranospinit

$$
\mathrm{H}_{3} \mathrm{O} \cdot\left\{\mathrm{UO}_{2} \cdot \mathrm{AsO}_{4}\right\}
$$

identisch sei. Für unsere eigenen Untersuchungen stand uns Originalmaterial von Schneeberg/Sachsen zur Verfügung. Die quantitative Analyse von sorgfältig ausgelesenen Kristallen ergab etwas niedrigere Werte für $\mathrm{UO}_{3}$ und $\mathrm{As}_{4} \mathrm{O}_{10}$ als sie $\mathrm{W}_{\text {INKLER }}{ }^{4}$ gefunden hatte, zusätzlich wurden beträchtliche Mengen $\mathrm{Bi}_{4} \mathrm{O}_{6}, \mathrm{PbO}$ und $\mathrm{P}_{4} \mathrm{O}_{10}$ gefunden. Der Trögerit besitzt ein hohes Kationen-Austauschvermögen von ca. $170 \mathrm{mval} / 100 \mathrm{~g}$ bei $110^{\circ} \mathrm{C}$ ge-

\footnotetext{
1 A. Weisbach, Neues J. Mineralog. 1871, 870.

2 H. Meixner, Chem. d. Erde 12, 433 [1939].

3 M. E. Mrose, Amer. Mineralogist 38, 1159 [1953].
}

trocknetem Material. Als Extremwerte beim Austausch gegen die Ammonium-Ionen einer wässerigen Ammoniumchloridlösung wurden an grobkristallinem Trögerit ca. $152 \mathrm{mval}$, an fein zerriebenem ca. $184 \mathrm{mval} / 100 \mathrm{~g}$ erhalten bei jeweils $140 \mathrm{Stdn}$. Verweildauer unter der Austauschlösung. Als austauschfähig gebundene Kationen wurden nachgewiesen:

$$
\mathrm{UO}_{2}{ }^{2+}, \mathrm{Pb}^{2+}, \mathrm{Ca}^{2+}, \mathrm{Mg}^{2+} \text { und } \mathrm{H}_{3} \mathrm{O}^{+} \text {. }
$$

Das 'Mineral kann reversibel ins Kristallinnere Wasser aufnehmen. Die wasserreiche Form ist grünlich, die wasserfreie Form kanariengelb gefärbt. Im natürlichen Material liegen wasserhaltige und wasserfreie Bereiche vor. Dies erklärt wohl die Angabe von Goldschmidt ${ }^{5}$, daß der gelbe Trögerit mit grünem Zeunerit verwachsen sei. Die Abwesenheit von Zeunerit $\mathrm{Cu} \cdot x \mathrm{H}_{2} \mathrm{O} \cdot\left\{\mathrm{UO}_{2} \cdot \mathrm{AsO}_{4}\right\}_{2}$ in unserer

${ }^{4}$ C.. Winkler, J. prakt. Chem. 7, 7 [1873].

5 V. M. Goldschmidt, Z. Kristallogr., Mineralog., Abt. A 31, 468 [1899]. 
Probe trotz einzelner grüner Bereiche ergab sich daraus, daß Kupfer nur in Spuren enthalten war.

Bei der eigenen optischen Untersuchung ließ sich die Angabe von Goldschmid bestätigen, daß das Mineral unter gekreuzten Nicols uneinheitlich er-

\begin{tabular}{|c|c|c|c|c|c|}
\hline \multicolumn{4}{|c|}{$\begin{array}{c}\text { Analysen von WINKLER } \\
\text { (auf lufttrockenes Material bezogen) }\end{array}$} & \multicolumn{2}{|c|}{$\begin{array}{l}\text { Eigene Analyse (Mittelwert) } \\
\text { (auf bei } 210^{\circ} \text { C getrocknetes } \\
\text { Material bezogen) }\end{array}$} \\
\hline \multicolumn{4}{|c|}{$[\%]$} & & {$[\%]$} \\
\hline $\begin{array}{l}\mathrm{UO}_{3} \\
\mathrm{As}_{4} \mathrm{O}_{10} \\
\mathrm{H}_{2} \mathrm{O} \\
\mathrm{Bi}_{4} \mathrm{O}_{6} \\
\mathrm{CuO} \\
\mathrm{CoO}\end{array}$ & $\begin{array}{c}63,76 \\
19,64 \\
14,81 \\
- \\
- \\
-\end{array}$ & $\begin{array}{r}59,73 \\
17,39 \\
17,03 \\
0,74 \\
0,56 \\
\text { Spuren }\end{array}$ & $\begin{array}{r}59,30 \\
17,89 \\
17,81 \\
2,21 \\
- \\
1,45\end{array}$ & $\begin{array}{l}\mathrm{UO}_{3} \\
\mathrm{As}_{4} \mathrm{O}_{10} \\
\mathrm{P}_{4} \mathrm{O}_{10} \\
\mathrm{CaO} \\
\mathrm{MgO} \\
\mathrm{PbO} \\
\mathrm{Bi}_{4} \mathrm{O}_{6} \\
\mathrm{CuO} \\
\mathrm{CoO} \\
\mathrm{H}_{2} \mathrm{O} 210-1000^{\circ} \\
\quad \quad 110-210^{\circ} \\
\quad \text { lufttr. }-110^{\circ}\end{array}$ & $\begin{array}{c}65,17 \\
22,08 \\
1,20 \\
0,65 \\
0,25 \\
1,56 \\
8,79 \\
\text { Spuren } \\
\text { Spuren } \\
0,16 \\
1,71 \\
7,9\end{array}$ \\
\hline
\end{tabular}

Tab. 1. Analysen von Trögerit.

scheint. In der Plättchenebene sind die Kristalle z. T. in scheinbar isotrope Felder unterteilt, die in doppelbrechende übergehen. Zum Teil sind quasiisotrope und doppelbrechende Bereiche scharf gegeneinander abgegrenzt, z. T. miteinander vergittert. In den quasi-isotropen Bereichen ist $n_{\mathrm{y}, \mathrm{z}}=1,63$ in Übereinstimmung mit den Werten von LARSEN ${ }^{6}$ und $2 V_{\mathrm{x}}=8-10^{\circ}$. Die doppelbrechenden Bereiche entsprechen den wasserreichen Bereichen. An ihnen wurde entsprechend dem höheren Wassergehalt $n_{\mathrm{yz}}$ mit 1,617 niedriger gefunden. Der Öffnungswinkel $2 V_{\mathrm{x}}$ beträgt in diesen Bereichen ca. $51^{\circ}$. Bei den optischen Messungen mit der Einbettungsmethode sinkt in den wasserfreien, gelben Bereichen $n_{\mathrm{yz}}$ langsam ab, während $2 V_{\mathrm{x}}$ dagegen ansteigt. Mit Einbettungs-Flüssigkeiten, welche Wasserspuren enthalten, erfolgt eine besonders rasche Änderung entsprechend der Wasseraufnahme ins Kristallinnere ${ }^{6 \mathrm{a}}$.

Die röntgenographische Untersuchung durch Drehkristall- und W e i ß e n b e r g - Aufnahmen mit CuK $\alpha$-Strahlung ergab, daß der Trögerit zur eindimensionalen innerkristallinen Quellung befähigt ist und deshalb zur Gruppe der Uranglimmer gehört ${ }^{7}$.

Bei den lufttrockenen gelben Kristallen waren die Gitterstörungen meistens so stark, daß die Aufnah-

6 E. Larsen, US Geol. Survey Bull. 679.

6a Vgl. hierzu die ähnlichen Beobachtungen bei quellungsfähigen Silikaten: C. W. Correns u. M. Mehmel, Z. Kristallogr., Mineralog. Petrogr., Abt. A 94, 337 [1936]; E. men nur schlecht auswertbar waren. Es gelang jedoch, einige ungestörte Kriställchen zu finden. Mit diesen ergaben sich die Gitterkonstanten:

$$
\begin{aligned}
a=b & =2 \cdot 6,9_{9}=13,9_{8} \AA, \\
c & =4 \cdot 8,4_{8}=33,9_{2} \AA
\end{aligned}
$$

mit (00l) nur für $\mathrm{l}=4 \mathrm{n}$ und (hk0) nur für $\mathrm{h}+\mathrm{k}=2 \mathrm{n}$. Die beobachteten Auslöschungen führen zu der Raumgruppe $\mathrm{D}_{4}^{4}-\mathrm{P} 4_{1} 2_{1} 2 \mathrm{bzw} . \mathrm{D}_{4}^{8}-\mathrm{P} 4_{3} 2_{1} 2$.

Zur Verdoppelung der a- und bAchse führten allerdings nur einige sehr schwache, diffuse Gitterstäbe in den W e i ß e n b e r g - Aufnahmen um [010].

Als Gitterkonstante für das bei $210^{\circ} \mathrm{C}$ getrocknete, wasserfreie $\mathrm{Mi}$ neral ergaben sich

$$
\begin{aligned}
a=b & =7,0_{4} \AA . \AA, \\
c & =8,3_{9} \AA .
\end{aligned}
$$

Die Kristalle wiesen durchwegs starke Gitterstörungen auf. Nur die Interferenzfolgen (h00), (0k0), (00l), (10l), (30l) und (50l) erwiesen sich als scharf. Die Gitterstäbe (20l), (40l), (60l), (22l), (42l), (62l) usw. zeigten nur zusammenhängende, zu größeren $\vartheta$-Winkeln hin langsam abklingende Schwärzungen. Als gesetzmäßige Auslöschung waren zu erkennen: (h00) nur mit $\mathrm{h}=2 \mathrm{n},(0 \mathrm{k} 0)$ nur mit $\mathrm{k}=2 \mathrm{n}$ und $00 \mathrm{l}$ nur mit $\mathrm{l}=2 \mathrm{n}$.

Für das mit Wasser bei $20^{\circ} \mathrm{C}$ gesättigte Mineral wurden die Gitterkonstanten

$$
\begin{aligned}
& a=7,1_{5} \AA, \\
& b=6,9_{9} \AA, \\
& c=11,2 \AA, \\
& \beta \approx 95^{\circ}
\end{aligned}
$$

erhalten. Wegen der geringen Anzahl der beobachteten Interferenzen und der starken Gitterstörungen sind die Dimensionen dieses Elementarkörpers aber nicht sehr genau. Keiner dieser drei Elementarkörper läßt sich einfach mit den alten, makroskopischen Achsenverhältnissen von $\mathrm{ScHRAUF}^{8}$ in Einklang bringen. Vielleicht sind die Achsenverhältnisse von Schrauf an einer der Quellungsstufen ermittelt, von welchen wir infolge der außerordentlich großen Gitterstörungen bisher noch keine auswertbaren W e i -

Maegdefrau u. U. Hofmann, Z. Kristallogr., Mineralog. Petrogr., Abt. A 98, 299 [1937]; R. E. GRIM u. W. F. Bradley, J. Amer. ceram. Soc. 23, 242 [1940].

7 A. Weiss u. U. Hofmann, Z. Naturforschg. 7 b, 362 [1952]. 
ßenberg-Aufnahmen erhalten konnten. In den Pulverdiagrammen ließen sich solche Quellungsstufen aber an den Schichtabständen von ca. 9,6 $\AA$ und 10,2 $\AA$ sicher nachweisen.

Die Gitterdimensionen, die chemische Zusammensetzung, das Kationen-Austauschvermögen und die

$$
\underbrace{\left\{\left(\mathrm{UO}_{2}\right)_{0,54} \mathrm{~Pb}_{0,07} \mathrm{Ca}_{0,11} \mathrm{Mg}_{0,06} \mathrm{H}_{3} \mathrm{O}_{0,08}\right\}^{1,64+}+x \mathrm{H}_{2} \mathrm{0}}_{\begin{array}{c}
\text { Wasserschicht mit austauschfähig } \\
\text { gebundenen Kationen }
\end{array}}
$$

Die Strukturformel wurde einmal aus dem Volumen der Elementarzelle, der chemischen Zusammensetzung, der Dichte und der Art und Menge der austauschfähig gebundenen Kationen errechnet. Sie wurde unabhängig davon auch aus der chemischen Zusammensetzung und dem Ionen-Austauschvermögen errechnet mit der Voraussetzung, daß die Summe von $\mathrm{AsO}_{4}{ }^{3-}$ und $\mathrm{PO}_{4}{ }^{3-}$ in der Uranglimmer-Schicht $=1,00$ ist.

Schwierigkeiten bereitete lediglich die Einordnung des Wismuts. Nach der analytischen Untersuchung liegt es wahrscheinlich in der Oxydationsstufe +3 vor. Beim Kationenaustausch konnte Wismut in der Gleichgewichtslösung nicht nachgewiesen werden. Es ließen sich aber auch keine durch eine Hydrolyse der bei einem eventuellen Austausch von Wismut entstehenden
Fähigkeit zur innerkristallinen Quellung ${ }^{7}$ lassen den Trögerit eindeutig in die Gruppe der Uranglimmer einreihen. Unter Berücksichtigung der Dichte von $3,9_{5}-4,1_{2}$ für das bei $210^{\circ} \mathrm{C}$ getrocknete Material ergibt sich als wahrscheinliche Strukturformel für den Trögerit:

$$
\underbrace{\left\{\left(\mathrm{UO}_{2}\right)_{0,82} \stackrel{+3}{\mathrm{Bi}}_{0,18}\left(\mathrm{AsO}_{4}\right)_{0,92}\left(\mathrm{PO}_{4}\right)_{0,08}\right\}^{0,82-}}_{\text {Uranglimmer-Schicht }}, .
$$

wismuthaltigen Gleichgewichtslösung freiwerdenden $\mathrm{H}_{3} \mathrm{O}$-Ionen nachweisen. Die $\mathrm{H}_{3} \mathrm{O}$-Ionen wurden erst beim Austausch in einer neutralen Ca-Acetat-Lösung frei. Da bei der optischen Untersuchung andererseits keine wismuthaltigen Verunreinigungen, wie z. B. Walpurgin, der z. T. mit dem Trögerit gemeinsam vorkommt, festgestellt werden konnten, erscheint die Einreihung in die Uranglimmer-Schichten an Stelle von $\mathrm{UO}_{2}$ sinnvoll.

Man könnte auch annehmen, $\mathrm{da} \beta$ nicht $\mathrm{Bi}^{3+}$-Ionen, sondern Bismutyl-Ionen $\mathrm{BiO}^{+}$an Stelle der Uranylionen in der Uranglimmer-Schicht sitzen. Diese Annahme fände unter anderem eine Stütze in der Analyse des wismuthaltigen Trögerits von $\mathrm{W}_{\text {INKLER }}{ }^{4}$, aus der sich dann die Strukturformel

$$
\underbrace{\left\{\left(\mathrm{UO}_{2}\right)_{0,391} \mathrm{Co}_{0,124}\right\}^{1,030+}+x \mathrm{H}_{2} \mathrm{O}}_{\begin{array}{c}
\text { Wasserschicht mit austauschfähig } \\
\text { gebundenen Kationen }
\end{array}}
$$

ergäbe.

Eine derartige Deutung unserer Analysenergebnisse ist jedoch nicht im Einklang mit dem experimentell bestimmten Kationen-Austauschvermögen, welches mehr dafür spricht, daß die Uranglimmer-Schichten durch den Wismutgehalt eine geringere negative Überschußladung erhalten als bei idealer Zusammensetzung oder gar beim Einbau von $\mathrm{BiO}^{+}$an Stelle von $\mathrm{UO}_{2}{ }^{2+}$.

Die im Gegensatz zu den anderen bisher näher untersuchten Uranglimmern in allen Quellungsstufen sehr viel stärker ausgeprägten Gitterstörungen und die begierige Wasseraufnahme unter gleichzeitiger innerkristalliner Ordnung, auch nach dem Erhitzen auf $200^{\circ} \mathrm{C}$, sprechen für diese niedrigere Schichtladung und sind mit den Erfahrungen bei der innerkristallinen Quellung von Mineralen der Montmorillonit-Vermikulit- und Glimmergruppe ${ }^{9}$ gut im Einklang.

$\mathrm{Da} \beta$ die Formel des Trögerits von der idealen $\mathrm{Zu}$ sammensetzung $\mathrm{UO}_{2}+x \mathrm{H}_{2} \mathrm{O}\left\{\mathrm{UO}_{2} \mathrm{AsO}_{4}\right\}_{2}$ abweicht, wird auch durch die ausgeprägte Überstruktur in der $\mathrm{a}-\mathrm{b}$-Ebene beim lufttrockenen Trögerit gestützt.

8 A. Schrauf, Z. Kristallogr., Mineralog. Petrogr., Abt. B 2, 185 [1872].

\section{$\underbrace{\left\{\left(\mathrm{UO}_{2}\right)_{0,938} \mathrm{BiO}_{0,062}\left(\mathrm{AsO}_{4}\right)_{1,000}\right\}^{1,062-}}$ \\ Uranglimmer-Schicht}

Der beim Umtausch der austauschfähigen Kationen gegen $\mathrm{NH}_{4}^{+}$-Ionen einer wässerigen $\mathrm{NH}_{4} \mathrm{Cl}$ Lösung entstehende „Ammonium-Trögerit“ erwies sich als ähnlich mit einem synthetisch hergestellten Ammoniumuranylarsenat, wenn man von den Gitterstörungen und der geringeren Schichtladung absieht.

Beim Austausch der Kationen gegen große Alkylammonium-Ionen wird der Trögerit wie die anderen Minerale der Uranglimmer-Gruppe organophil und kann in den verschiedensten Flüssigkeiten quellen ${ }^{10}$. So beträgt z. B. der Schichtabstand eines „n-Stearylammonium-Trögerit" nach der Trocknung bei $65^{\circ} \mathrm{C} 27,6 \AA$, unter einer wässerigen Sterarylammoniumchlorid-Lösung 33,0 $\AA$ und unter n-Dodecylalkohol z. B. $47,0 \AA$.

9 A. Weiss, A. Mehler u. U. Hofmann, Z. Naturforschg. 11 b, 435 [1956] ; A. Weiss, G. Кoch u. U. Hofmann, Ber. dtsch. keram. Ges. 32, 12 [1955].

10 A. Weiss, K. Hartl u. U. Hofmann, im Druck. 